Article

\title{
Improvement of sensitivity and detectability of the three-coil wear debris detection sensor using LC resonance method and modified lock-in amplifier
}

\author{
Ran Jia ${ }^{1}$, Biao Ma ${ }^{1}$, Changsong Zheng ${ }^{1 *}$, Liyong Wang ${ }^{2}$, Qiu Du ${ }^{1}$, Kai Wang ${ }^{1}$ \\ 1.School of mechanical engineering, Beijing institute of technology, Beijing 10081, P.R.China; \\ 2.The ministry of education key laboratory of modern measurement and control technology, Beijing Information \\ Science and Technology University, Beijing 100192, P.R.China \\ * Correspondence: Tel.: +86-010-68918636
}

\begin{abstract}
That how to improve the sensitivity and the detection ability of the Large-caliber sensor is critical to satisfy the requirement of heavy vehicle wear condition monitoring. In this paper, we introduced the LC resonance principle to the design of sensor with large flow channel $(7 \mathrm{~mm})$. The resonant exciting coil increases the impedance change of the exciting circuit caused by wear particles which magnify the current difference between the two exciting coils and improve the sensitivity of the sensor. The resonant induction coil greatly suppresses the interference signals and magnifies the weak induced electromotive force which is beneficial to enhance the detection ability. In order to boost the detectability for different materials particles, a phase controlled variable-frequency exciting system (PCVFES) was adopted to automatically switch the exciting frequency between $284 \mathrm{kHz}$ for ferromagnetic particles and $420 \mathrm{KHz}$ for non-ferromagnetic particles. For the weak signal detection, based on the essential characteristic of the signal, we apply a simpler method that modified lock-in amplifier (MLIA) rather than the conventional algorithms (Wavelet transform, EMD). Results show that using these methods the sensitivity and the detection ability of the sensor are significantly improved and the $75 \mu \mathrm{m}$ iron particles and $220 \mu \mathrm{m}$ copper particles were successfully detected which realizes the initial abnormal wear monitoring for the large flow project.
\end{abstract}

Key words: wear debris detection; resonance method; sensitivity; phase controlled variable-frequency exciting system (PCVFES); modified lock-in amplifier (MLIA)

\section{Introduction}

The wear is the most common fault modality in mechanical equipment and also is one of the major obstacles affecting the normal operation of machinery and equipment. With the development of modern industry, the demands about continuity of production and the reliability of mechanical equipment continue to increase, therefore, that the ability to precisely monitor the wear condition of mechanical equipment and to prevent serious malfunction caused by excessive wear gradually show its significance. The wear debris detection sensor is thus an effective tool for wear condition monitoring ${ }^{[1]}$ and starts to show its great vitality because of the high detection efficiency, veracity and the ability to timely reflect the wear state changing process of equipment.

At the present, some research institutions have manufactured various kinds of wear debris detection sensor based on different physical principles mainly including optics (light scattering and diffraction), ultrasonic, electrics (capacitance, resistance and inductance) and image ${ }^{[2]}$. By comparing these sensors' properties, it is shown that the optical wear particle detection sensor is easily affected by the bubbles in the oil and gives false negative results. And the ultrasonic wear particle detection sensor is easily disturbed by background noise and has lower 
temperature stability. These drawbacks greatly limit the use of this type of sensor under the complicated operating conditions and extreme environment. The image type abrasive detection sensor has very complex structure which judge the material properties of particles based on the color, so it is easily influenced by the inhomogeneity of light, but actually, the most significant hardship is that the detection speed and precision are closely related to the image processing algorithm, and it is difficult to guarantee high test accuracy and real time synchronously. While for the electromagnetic wear debris detection sensor, with the advantages of simple and reliable structure, good temperature stability and strong ability of anti-background noise it has shown more and more applications in the wear monitor field.

For the study of this kind of sensor, institutions carried out various researches from different perspectives. The first is that sensors with different coil structure were studied. According to the amounts of the coils in the sensor, that can be divided into three kinds that single-coil, double-coil and three-coil abrasive detection sensor ${ }^{[3-8]}$, the advantages and disadvantages are listed in the ${ }^{[8]}$. The first two kinds of sensors which have lower detection sensitivity due to its structural restrictions are gradually showing marginalization. While the three-coil wear particle detection sensor is widely studied ${ }^{[6-8]}$. Besides that the sensors with different coil shapes were also studied which mainly includes planar coil structure and spiral coil structure ${ }^{[2,9,10]}$. The second important research direction is that sensors with micro channel structure which outstanding characteristic is that the inner diameter of the channel is often smaller than $1 \mathrm{~mm}^{[11-14]}$ but have high sensitivity. Generally this kind of sensor can successfully detect up to 20um ferromagnetic particles and 55um non-ferromagnetic particles, but because of the limitation of its maximum flow rate, it is difficult to meet the requirements of the large flow engineering. The third is study on the recognition method of the weak inductive electromotive force. In this area the most popular method is the threshold method. With this approach, a metallic particle signature is confirmed if the amplitude of the sensor output signal is above a preset threshold value. This is obviously valid only if the signal-to-noise ratio (SNR) of the signal is sufficiently high $^{[15]}$. While in the real sensor, especially for large-aperture sensor, the SNR is really small. Except that the other two methods are also commonly used that are method based on wavelet transform and method based on empirical modal decomposition (EMD) ${ }^{[15-20]}$. These two methods use the similar idea that decompose the original signal into multilayer according to the different signal components and filter out the invalid components and finally extract the weak induced electromotive force. These methods indeed improve the detection effect of the weak signals to some extent, but in real practice, the solution of the algorithm has certain requirements for the computing power of the equipment and hard to ensure real-time processing of the signal. Besides that, it is difficult for the algorithm to adaptively adjust its own parameters according to the particle size and the particle movement speed, so the weak signal extraction method still need further study.

The research in this paper is driven by the online wear state monitoring requirements for heavy vehicles and studied the method of improving the sensitivity and the weak signal extraction method for the large flow (allowable maximum flow $\geq 28 \mathrm{~L} / \mathrm{min}$ ) sensor. In order to improve the sensitivity of the sensor, the parallel resonance principle was introduced to the traditional three-coil wear particles detection sensor. Through replace the impedance change of coil caused by debris by the impedance alteration of the LC circuit around the resonant frequency the sensitivity of the sensor can be significantly improved. For the weak signal extraction method, according to the characteristic of the weak induced electromotive force that has an equal frequency with the reference signal, a measurement system based lock-in amplifier (LIA) was designed to extract the effective signal from the noise, Because the best detection frequency for ferromagnetic and non-ferromagnetic particles is different, so a phase controlled variable-frequency exciting system (PCVFES) was joined to the measurement system. This signal detection system is all implemented by hardware and not need any additional mathematical algorithms, so that greatly reduced the amount of computation of the system and more satisfied the requirements of real-time and reliability of the vehicle wear state detection project. 


\section{Device description}

\subsection{The novel structure and parameters of the sensor}

Fig.1a illustrates the novel structure of the three-coil wear particles detection sensor that contains a shell made by 304 steel, an electromagnetic shield layer, a ceramic coil frame, two exciting coils, an inductive coil, and the corresponding resonance capacitor. In the sensor, The two reverse exciting coils with same sine wave current enwind on the both sides of the coil frame made by machinable ceramics (thermal conductivity $\lambda=1.71 \mathrm{~W} / \mathrm{m} \cdot \mathrm{k}$ ), and the inductive coil lies in the middle of it. The ceramic was adopted on the coil frame because of both its magnetic insulation and low thermal-conductivity to reduce the effect of the heat of the high temperature oil in the flow channel on the detection sensitivity. The electromagnetic shield layer is made of the combination of copper foil and ferrite to decrease the interference of external electromagnetic fields. Meanwhile, the resonance capacitances are connected to the exciting and inductive coils to form LC parallel resonance circuit and make these coils working on resonance state simultaneous. And it needs to be emphasized that for the best measurement, the resonance frequency of exciting coil and inductive coil must be consistent.

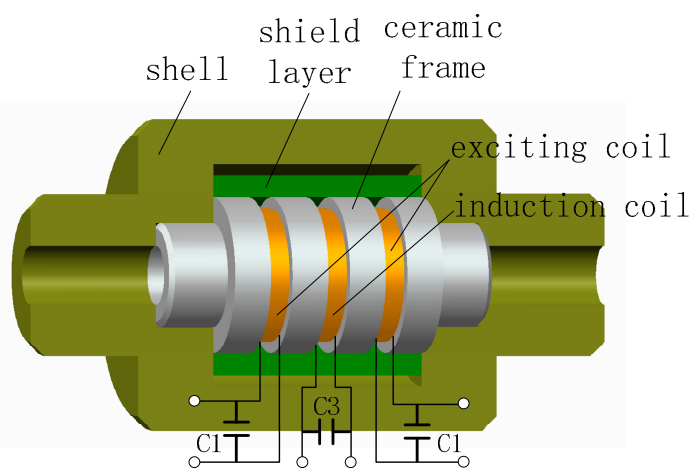

(a)

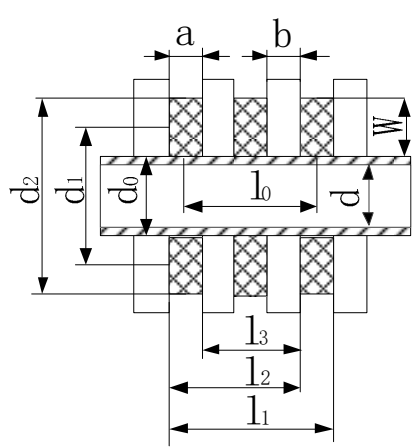

(b)

Fig.1 structure of the sensor

The structure of the core component of the sensor is shown at Fig.1b. Through the final optimization of previous work ${ }^{[21]}$ the structure parameters of the core component were obtained. And the optimized structure information is listed in Table 1. In order to meet the flow demands of practical engineering application, especially for some significant mechanical devices such as the airplanes, vehicles and boats; the inner diameter of this sensor was designed to $7 \mathrm{~mm}$ which assure the maximum allowable flow can reach to $35 \mathrm{~L} / \mathrm{min}$. In the mechanical system, the size of wear debris range from $20 \mu \mathrm{m}$ to $50 \mu \mathrm{m}$ during the normal wear period, while in the initial abnormal wear stage the particle size increase to $50 \mu \mathrm{m}$ to $100 \mu \mathrm{m}$. As a consequence, in order to achieve effective monitoring of the abnormal wear stage, the sensor must be able to successfully detect $50-100 \mu \mathrm{m}$ particles. But with the increase of the diameter of flow channel, the sensitivity and the detection capability about micro metal particles decreased. Therefore, that improving the sensitivity and the weak signal detection capability is significant process in the engineering process of such sensors.

Table 1. Parameters of the sensor

\begin{tabular}{ccccc}
\hline Width of coil $a$ & Coil spacing $b$ & $\begin{array}{c}\text { Inside channel } \\
\text { diameter } d\end{array}$ & $\begin{array}{c}\text { Outside channel } \\
\text { diameter } \mathrm{d}_{0}\end{array}$ & $\begin{array}{c}\text { Exciting coils } \\
\text { spacing } \mathrm{I}_{0}\end{array}$ \\
\hline $2 \mathrm{~mm}$ & $2.5 \mathrm{~mm}$ & $7 \mathrm{~mm}$ & $10 \mathrm{~mm}$ & $9 \mathrm{~mm}$ \\
\hline
\end{tabular}




\begin{tabular}{cccc}
\hline & Turns of exciting & Turns of inductive & Exciting Current \\
Wire diameter $\mathrm{d}_{w}$ & coil $N_{e}$ & coil $N_{i}$ & $I_{0}$ \\
\hline $0.2 \mathrm{~mm}$ & 180 & 195 & $50^{-3} \sin \left(\omega_{0} \mathrm{t}+\varphi_{0}\right)$ \\
\hline
\end{tabular}

The inner magnetic field of the sensor $\mathrm{B}_{\mathrm{sx}}$ is composed by the magnetic field of the two exciting coils. The magnetic induction along the axis of single coil $\mathrm{B}_{\text {sol }}(\mathrm{x}, \mathrm{y})$ is shown at ${ }^{[21]}$, so the $\mathrm{B}_{\mathrm{sx}}$ can be found in equation (1). Using the above optimized parameters to calculate the distribution of the magnetic field in the sensor, we can obtain that the maximum value of $\mathrm{B}_{\mathrm{sx}}$ is $0.035 \mathrm{~T}$ located near the middle of the exciting coil. When wear particles enter into the sensor, the magnetic field in the exciting coil will be changed $\Delta \mathrm{B}(\mathrm{x}, \mathrm{y})$, so the alteration of the magnetic flux caused by particles is as equation (2), and the induced electromotive force output by the induction coil can be described as Equation (3). So the magnitude of the induced electromotive force is related to the change rate of the product of the inductance and current in the coil. For a particular coil, the effect of metal wear particles on coil inductance is very weak, so the main method of increasing the induced electromotive force and improving sensitivity of the sensor is to enlarge the current alteration in the exciting coil caused by wear particles.

$$
\begin{gathered}
\mathrm{B}_{\mathrm{sx}}=\mathrm{B}_{s o l}(\mathrm{x}, \mathrm{y})-\mathrm{B}_{s o l}\left(\mathrm{x}+\mathrm{1}_{0}, \mathrm{y}\right) \\
\Delta \phi=\int_{s} \Delta \mathrm{B}(\mathrm{x}, \mathrm{y}, \mathrm{t}) \mathrm{ds} \\
\mathrm{E}=\mathrm{N} \frac{\Delta \phi}{\Delta \mathrm{t}}=\mathrm{N} \frac{\Delta(L \times \mathrm{I})}{\Delta \mathrm{t}}
\end{gathered}
$$

The original coil inductance of the sensor in the empty situation can be written as equation (4). And the magnetic coupling between the two exciting coils leads to mutual inductance effect which will change the inductance of the exciting coils. The mutual inductance can be expressed as equation (5)

$$
\begin{gathered}
\mathrm{L}=5.4 \frac{\mu_{0}}{4 \pi} \frac{\mathrm{N}^{2} \mathrm{~d}_{1}^{2}}{\sqrt{a \mathrm{w}}} \\
M=\frac{\pi \mu_{0}}{8} N^{2} \frac{d_{2}^{2}}{a^{2}}\left(k_{1} F_{1}-2 k_{2} F_{2}+k_{3} F_{3}\right)
\end{gathered}
$$

Where: $\mu_{0}=4 \pi \times 10^{-7}$ is permeability of vacuum, $\mathrm{N}$ is turns per coil, $\mathrm{d}_{1}$ is the mean diameter of the coil, $\mathrm{d}_{2}$ is the outside diameter of the coil, $a$ is the width of coil, $w$ is thickness of coil, and $k_{i} \mathrm{~F}_{i}$ as follows:

$$
\begin{aligned}
& k_{i}=\sqrt{\left(d_{2} / 2\right)^{2}+l_{i}^{2}} \\
& F_{i}=-\frac{1}{5} \lambda_{i}^{4}-\frac{1}{2^{6}} \lambda_{i}^{2}+1 \\
& \lambda_{i}=d_{2} /\left(2 k_{i}\right)
\end{aligned}
$$

So the equivalent inductance of single exciting coil is $\mathrm{L}_{\mathrm{q}}=\mathrm{L}-\mathrm{M}$. Based on the calculation, the theoretical inductance of the exciting coil and inductive coil are $300.8 \mu \mathrm{H}$ and $408.6 \mu \mathrm{H}$ respectively. And the mutual inductance between the two exciting coils is $\mathrm{M}=45.586 \mu \mathrm{H}$. In experiment, we measured these inductances by TONGHUI digital electric bridge tester (TH2821B) and found that the real equivalent inductance of exciting coil is $313.5 \mu \mathrm{H}$ and the inductance coil is $427.2 \mu \mathrm{H}$. The mutual inductance of the two exciting coils can be obtained that $\mathrm{M}=43.3 \mu \mathrm{H}$. 


\subsection{The method of improving sensitivity}

Based on the equation (3), that increase the current change in the exciting coil when wear debris enter into the sensor can improve the induced electromotive force and improving sensitivity of the sensor. So in order to boost the sensitivity and validity of the sensor with large aperture, The LC parallel resonance method was introduced to the traditional three-coil wear particles detection sensor. By ensuring the excitation coil in the resonance state to enlarge the current in the exciting coil and make the metal particles produce a stronger magnetization or eddy current effect to increase the impact on the external magnetic field. And because of the impedance change of the LC parallel resonant circuit caused by wear particles is larger than impedance change of the inductance coil, so there will be a greater current difference between the two excitation coils and then the induction coil will produce greater magnetic flux changes and output a larger induced electromotive force. At the same time, the resonant induction coil will magnify the weak induced electromotive force to a certain degree, and another important role is that filter the noises mixed in the signal, that is very helpful to improve the sensitivity and detection ability about micro particles of the sensor.

In order to demonstrate the mechanism of improving sensitivity using LC parallel resonance method, the sensitivity analysis of the sensors with resonance method and non-resonance method were conducted. The schematic diagram of the two sensors is shown in Fig.2, where L1 and L2 represent the two exciting coils, L3 is the induction coil, $\mathrm{r} 1 \mathrm{r} 2$ and $\mathrm{r} 3$ are the internal resistance of coils. The Fig.2a is a circuit diagram without resonance method that used in our prior work ${ }^{[21]}$, and Fig.2b is circuit diagram using LC parallel resonance mechanism where $\mathrm{C} 1 \mathrm{C} 2$ and $\mathrm{C} 3$ are the resonant capacitances for corresponding coils. In all circumstance, the exciting signal adopted the unique sinusoidal current source $\left(I_{0}=50-3 \sin \left(2 \pi f_{0} t\right) A\right)$ where $f_{0}$ is the resonance frequency.

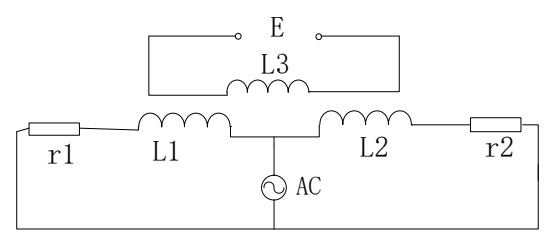

(a)

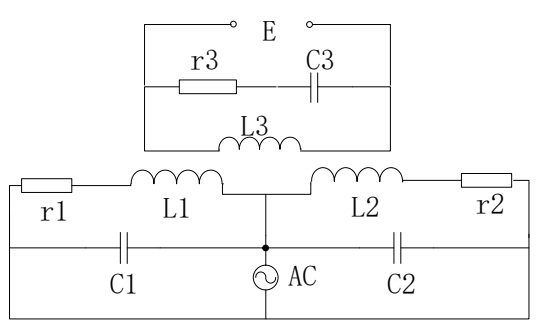

(b)

Figure 2 Circuit diagram of the sensor

When no particle enters into the sensor, the impedance of the unilateral exciting circuit is shown in equation (6), where $L_{q}=270.2 \mu \mathrm{H}$, the internal resistance of exciting coil $\mathrm{r} 1=\mathrm{r} 2=4 \Omega$. The excitation frequency $\mathrm{f}_{0}$ was set to $284 \mathrm{kHz}$ and $420 \mathrm{kHz}$ respectively for wear particles of different materials. As all the excitation coils in sensor b are working in the resonant state, so the resonant condition must be satisfied as equation (7).

$$
\begin{gathered}
Z_{a}=j \omega L_{q}+r \\
Z_{b}=\frac{\left(j \omega L_{q}+r\right) \frac{1}{j \omega C}}{\left(j \omega L_{q}+r\right)+\frac{1}{j \omega C}}=\frac{\left(j \omega L_{q}+r\right)}{(j \omega \mathrm{C} r)} \\
f=\frac{1}{2 \pi \sqrt{L C}}
\end{gathered}
$$

At this circumstance, the internal resistance of coils can be ignored and we can obtain that the impedance of exciting circuit of sensor $b$ is much larger than sensor a as equation (8) which also means that a slight change of the coil inductance result from wear particles will result in a larger impedance change in the exciting circuit of sensor $b$ than sensor a and then produces a greater current change in the excitation coil. 


$$
\left|\mathrm{Z}_{b}\right|=\frac{\omega^{2} \mathrm{~L}^{2}}{r} \square\left|\mathrm{Z}_{a}\right| \approx \omega \mathrm{L}
$$

When no particles enter into the sensor, the current in the excitation coil of different sensors are given by equation (9). From the equation, we note that the current in the coil of sensor b is closely related to the exciting frequency. Fig. 3 shows the current difference of exciting coil between the two sensors (resonance frequency f0 is $284 \mathrm{kHz}$, capacitance $\mathrm{C} 1=\mathrm{C} 2=2.0 \mathrm{nF}$ ). We can find that when the exciting frequency is equal to the resonance frequency, the current in exciting coil of sensor $\mathrm{b}$ can reach to $1.7 \mathrm{~A}$ which is much larger than that of sensor a (25mA). Because the magnetic flux can be expressed with $\Phi=\mathrm{LI}$, so in the resonance state, there's more magnetic flux in the sensor $b$ which is beneficial to boost the magnetization or Eddy current effect of the wear particle and enhance debris detectability.

$$
\begin{aligned}
& \left|\mathrm{I}_{\mathrm{a}}\right|=\left|\mathrm{I}_{0}\right| / 2 \\
& \left|\mathrm{I}_{\mathrm{b}}\right|=\left|\frac{\mathrm{I}_{0} * Z_{b}}{2 *\left(r+j \omega L_{q}\right)}\right|=\frac{\omega^{3} L_{q}}{r}\left|\mathrm{I}_{\mathrm{a}}\right|
\end{aligned}
$$

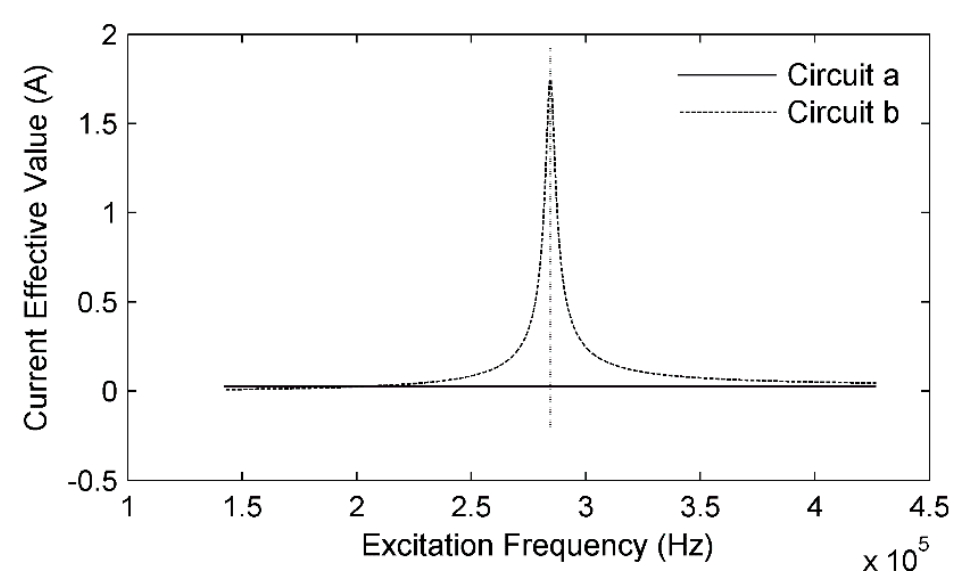

Fig. 3 Current in the exciting coil at different frequency

When some debris access the sensor, the inductance disturbance of the coil will be generated, and for ferromagnetic particles $\Delta \mathrm{L}>0$ because of magnetization while for non-ferromagnetic particles $\Delta \mathrm{L}<0$ because of Eddy current effect. So the inductance of excitation coil alters to $\mathrm{L}_{\mathrm{t}}=\mathrm{L}_{\mathrm{q}}+\Delta \mathrm{L}$. As a consequence, the impedance of exciting circuit can be modified to be:

$$
\begin{aligned}
& \mathrm{Z}_{a}^{\prime}=\mathrm{Z}_{a}+\Delta \mathrm{Z}_{a}=j \omega L_{t}+r \\
& \mathrm{Z}_{b}^{\prime}=\mathrm{Z}_{b}+\Delta \mathrm{Z}_{b}=\frac{\frac{r}{(\omega c)^{2}}-j \frac{1}{\omega c}\left[r^{2}+\left(\omega L_{t}\right)^{2}-\frac{L_{t}}{C}\right]}{r^{2}+\left(\omega L_{t}-\frac{1}{\omega c}\right)^{2}}
\end{aligned}
$$

In order to characterize the difference of sensitivity between the two types of sensor, we take ferromagnetic wear debris as an example. The inductance change caused by ferromagnetic particle with radius ra is shown at equation (11),where 1 is the length of the coil.

$$
\Delta \mathrm{L}=\frac{(\sqrt{5}-1) \mu_{0} \mu_{\mathrm{r}} N^{2} r_{a}^{3}}{l^{2}}
$$

The inductance change of exciting coil caused by ferromagnetic particle of diameter range 0 to $700 \mu \mathrm{m}$ and the corresponding impedance change of the unilateral exciting circuit are shown as Fig4. In this analysis, we used exciting frequency $\mathrm{f} 0=100 \mathrm{kHz}$, resonance capacity $\mathrm{C} 1=\mathrm{C} 2=10.35 \mathrm{nF}$. It can be seen that the impedance change of sensor $b$ is more noticeable than just change in sensor a. For the sensor $a$, the impedance change due to a particle of 
$700 \mu \mathrm{m}$ in diameter is only $0.235 \Omega$, while for the sensor b, the change of impedance caused by a $528 \mu \mathrm{m}$ in diameter can reach to $3.99 \Omega$, Which reflect that the LC resonance mechanism can increase the sensor's sensitivity at some extent. And it is noteworthy that unlike the impedance linear increase with the coil inductance rise of sensor $\mathrm{a}$, the impedance alteration of sensor $\mathrm{b}$ takes on the non-linear characteristic and exhibits a peak value at $\mathrm{r} 1$ position. And after the r1, the impedance change decrease rapidly due to its severe impedance mismatch. That means that for the sensor $\mathrm{b}$ the effective detection range must limited to $[0, \mathrm{rl}]$ ( $\mathrm{r} 1=528 \mu \mathrm{m}, \Delta \mathrm{Z}=3.99 \Omega)$. But in order to further improve the detectability of small wear particles, some measures must be taken to decrease the value of $\mathrm{r} 1$. It is calculated that the resonance capacitor will greatly affect the position of r1. Fig.5 shows the impedance change curve of different capacitance of $10 \mathrm{nF}, 8 \mathrm{nF}, 6 \mathrm{nF}, 4 \mathrm{nF}, 2 \mathrm{nF}$. It can be seen that the various capacitance won't change the maximum value of impedance change, but move the impedance change curve to left which make the detection ability for micro-particle been dramatically improved. However, an excessively small capacity will lead to higher excitation frequency that will enhance the eddy current effect of metal particles and decrease the detectability about ferromagnetic particles, so in this sensor a capacitance of $2 \mathrm{nF}$ was chosen for ferromagnetic particle detection, and the corresponding exciting frequency is $\mathrm{f}_{0}=284 \mathrm{kHz}$. At this situation, the detection range was restricted to $[0,300 \mu \mathrm{m}]$ due to the nonlinear feature of the impedance change. The ferromagnetic particle which radius is larger than $r_{2}$ will be recognized as non-ferromagnetic particles due to the negative impedance change result from serious mismatch.

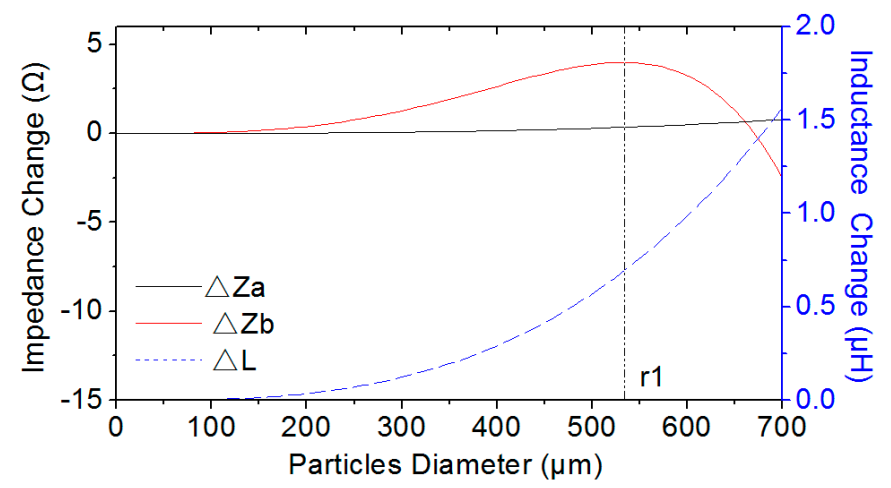

Fig.4 Alternation of inductance and impedance of the coil caused by wear particles

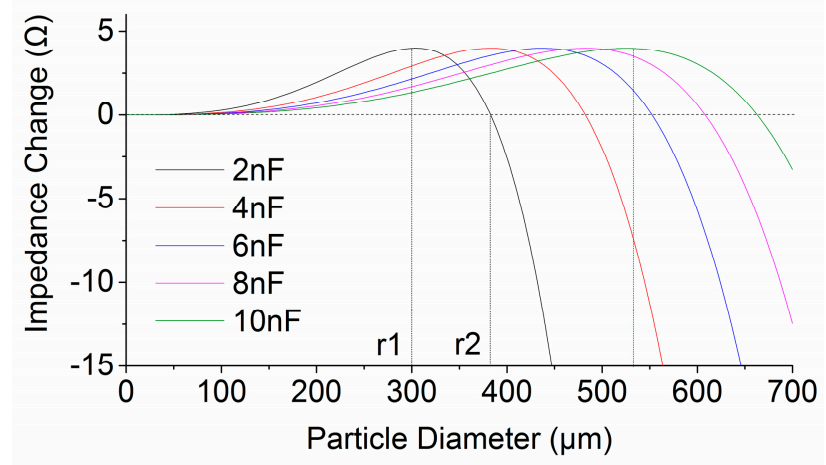

Fig.5 The influence of resonance capacitance on the impedance change

Because of the impedance change of the exciting circuit in a sensor, the current in the exciting coil will be also redistributed and produce current difference between the two excitation coils of the sensor as equation (12). Under the circumstance, that resonance capacitor equals to $2 \mathrm{nF}$ and the current differentials on both exciting coils of the two sensors are shown in the Fig.6. We also can obtain that the current difference of the two exciting coils in the sensor $\mathrm{b}$ which adopted LC parallel resonance principle is much larger than that of sensor a. The induced electromotive force output by the induction coil output can be expressed as equation (13), where $\lambda$ is a scattering 
coefficient which is related to the structure of the sensor. From the equation, we can know that a larger current change will lead to the increase of induced electromotive force output by the induction coil caused by the same particle and essentially improve the detection sensitivity of the sensor.

$$
\begin{aligned}
& \Delta \mathrm{I}_{\mathrm{a}}=\mathrm{I}_{0} * \mathrm{Z}_{\mathrm{a}}\left(\frac{1}{\mathrm{Z}_{1}+\Delta \mathrm{Z}}-\frac{1}{\mathrm{Z}_{2}}\right) \\
& \Delta \mathrm{I}_{\mathrm{b}}=\mathrm{I}_{0} * \mathrm{Z}_{\mathrm{b}}\left(\frac{1}{\mathrm{Z}_{\mathrm{L}}+\Delta \mathrm{Z}_{L}+r}-\frac{1}{\mathrm{Z}_{\mathrm{L}}+r}\right)
\end{aligned}
$$

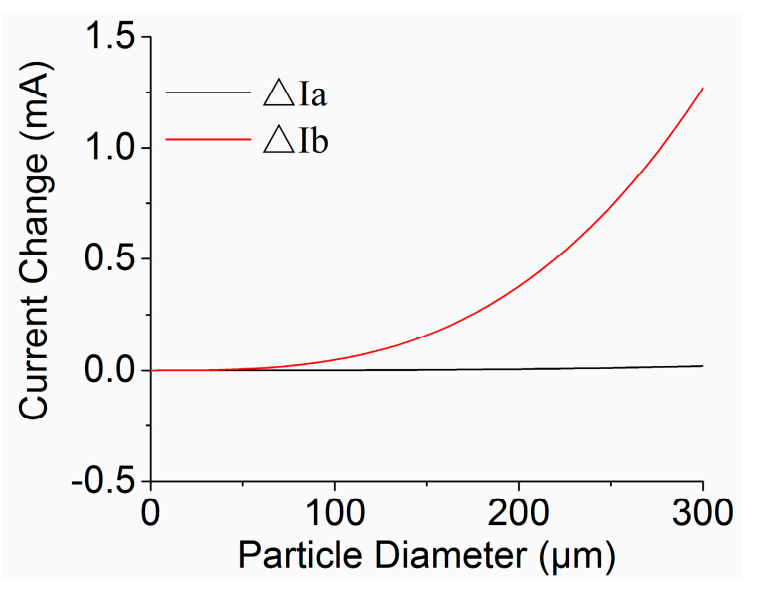

Fig.6 The current difference between the two exciting coils of the two sensors

$$
\mathrm{E}_{0}=\frac{\Delta \phi}{\Delta t}=\lambda \frac{(L+\Delta \mathrm{L})(I+\Delta \mathrm{I})-L I}{\Delta t}
$$

In order to detect the weak signal precisely, the induction coil is also required working in the resonance state. So a corresponding resonance capacity is also needed and the value of the capacity also meets the resonance condition as equation (7). It is noteworthy that the resonance frequency should equal to the exciting frequency and guarantee these three coils in the state of resonance simultaneously. That making the inductive coil working in resonance state will bring two obvious advantages that filter noises of non-resonance frequency and magnify induced electromotive force E0. The amplitude-frequency characteristics of the induction coil in the resonant state is in equation (14), where $\alpha_{v}(j \omega)$ is the amplitude ratio of a particular frequency component signal, $\mathrm{Q}$ is the quality factor of inductive coil as equation (15), $\omega_{0}$ is the inherent angular frequency. For the induction coil $\mathrm{L}=427.2 \mu \mathrm{H}$, $\mathrm{r}=4.36 \Omega, \mathrm{C}=0.74 \mathrm{nF}$ for $\mathrm{f}_{0}=284 \mathrm{kHz}, \mathrm{Q}=174.8$. In the output signal, there must contains noise signal and the effective induced electromotive force and generally, the noise signal has a wide spectrum while the valid signal is contained in the sinusoidal signal which has the same frequency with the excitation signal. Based on the amplitude-frequency characteristics, the amplitude ratio of the second harmonic signal is just 0.0038 . So the noise signal mixed in the induced electromotive force will be largely eliminated. Besides that the induction coil will magnify the weak induced electromotive force and the magnification is $\mathrm{Q}$, so the real output signal of the induction coil is $\mathrm{Eo}_{\mathrm{Q}} \mathrm{QE}_{0}$. As a consequence, this ability of signal filtering and amplification is very useful for particle detection.

$$
\alpha_{v}(j \omega)=\frac{1}{\sqrt{1+Q^{2}\left(\frac{\omega}{\omega_{0}}-\frac{\omega_{0}}{\omega}\right)^{2}}}
$$




$$
\mathrm{Q}=\frac{1}{r} \sqrt{\frac{L}{C}}
$$

For the detection of non-ferromagnetic particles, because the impact of eddy current effect on magnetic field is excessively weak, so that is more difficult to detect. In order to further improve the detection ability for non-ferromagnetic particles of the sensor, it is necessary to increase the excitation frequency to enhance the eddy current effect inside the non-ferromagnetic particles. After several experiments, the exciting frequency for non-ferromagnetic particles was set to $420 \mathrm{kHz}$, the corresponding resonant capacitor $\mathrm{C} 1=\mathrm{C} 2=0.9 \mathrm{nF}, \mathrm{C} 3=0.33 \mathrm{nF}$, $\mathrm{Q}=261$. Therefore, a variable-frequency excitation system was designed for the sake of requirements for the excitation frequency of particles of different materials.

\subsection{Signal excitation and detection method}

In this sensor, the exciting system and weak signal detection system are very important to the detection effect. In the exciting system, a higher exciting frequency is helpful to improve the sensitivity, especially for detection of non-ferromagnetic particles because of the more severe eddy current effect, while for ferromagnetic particles the excessive eddy current will decrease the inductance alteration of the coil caused by particle magnetization. So in this paper, a variable-frequency excitation system was designed which has two exciting frequency that $284 \mathrm{kHz}$ for ferromagnetic particles and $420 \mathrm{kHz}$ for non-ferromagnetic particles. The weak signal processing system takes the advantages of the valid signal characteristics that it has the same frequency and phase with the exciting signal. So that doesn't adopt any mathematical algorithm but a more simple and rapid method that the technology of lock-in amplifier which was used to suppress noises and promote useful signals several magnitudes. The block diagram of the signal exciting and detection system is shown at Fig.7 which can be divided into two parts that phase controlled variable-frequency exciting system (PCVFES) and the modified lock-in amplifier (MLIA). In the whole system, the square signal was used as the external exciting signal. Because of the frequency difference for ferromagnetic particles detection $(284 \mathrm{kHz})$ and non-ferromagnetic particles detection $(420 \mathrm{kHz})$, a frequency synthesizer was used to change the exciting frequency based on the signal phase. The output signal caused by ferromagnetic particles and non-ferromagnetic particles has the opposite phase, so we used the comparator to transform the sinusoidal wave to square signal and through the rising edge detection to judge whether the particle approaching to the exciting coil is ferromagnetic or not (cause the magnetic field expose to the outside of sensor core, so when a metal particle close to the exciting coil and not enter into it, a signal disturbance will start generating) and control the frequency synthesizer output exciting signal of different frequency. The amplifier was used to magnify the magnitude of the exciting signal to improve its drive power. In order to maintain the three coils are lying in the resonance state, a capacitance matcher was used to change the resonance capacitance according to the real exciting frequency. The weak signal detection system mainly adopted the modified Lock-in amplification system (MLIA) which reference signal frequency is also controlled by the frequency synthesizer. In the MLIA two 8th-orders Bessel-type low pass filter were adopted which cut-off frequency was set to $1.5 \mathrm{kHz}$ due to the facts that the amplitude of the signal is crucial important for the particle detection and the Bessel filter has the most flat amplitude response and the maximum frequency of the valid signal is $1.37 \mathrm{kHz}$. After that, a 5th-order Butterworth high pass filter was used with cutoff frequency $5 \mathrm{~Hz}$ to filter out the initial induced electromotive force caused by coil offset or magnetic field inhomogeneity. With this system, we can filter out the Gaussian noises and disturbance signal caused by magnetic field inhomogeneity of the two exciting coils which are much larger than valid induced electromotive force. This system can be fully implemented by hardware and avoid a lot of mathematical operations on the computer which greatly reduce the complexity of the system and more satisfy with the real-time requirements of equipment. 


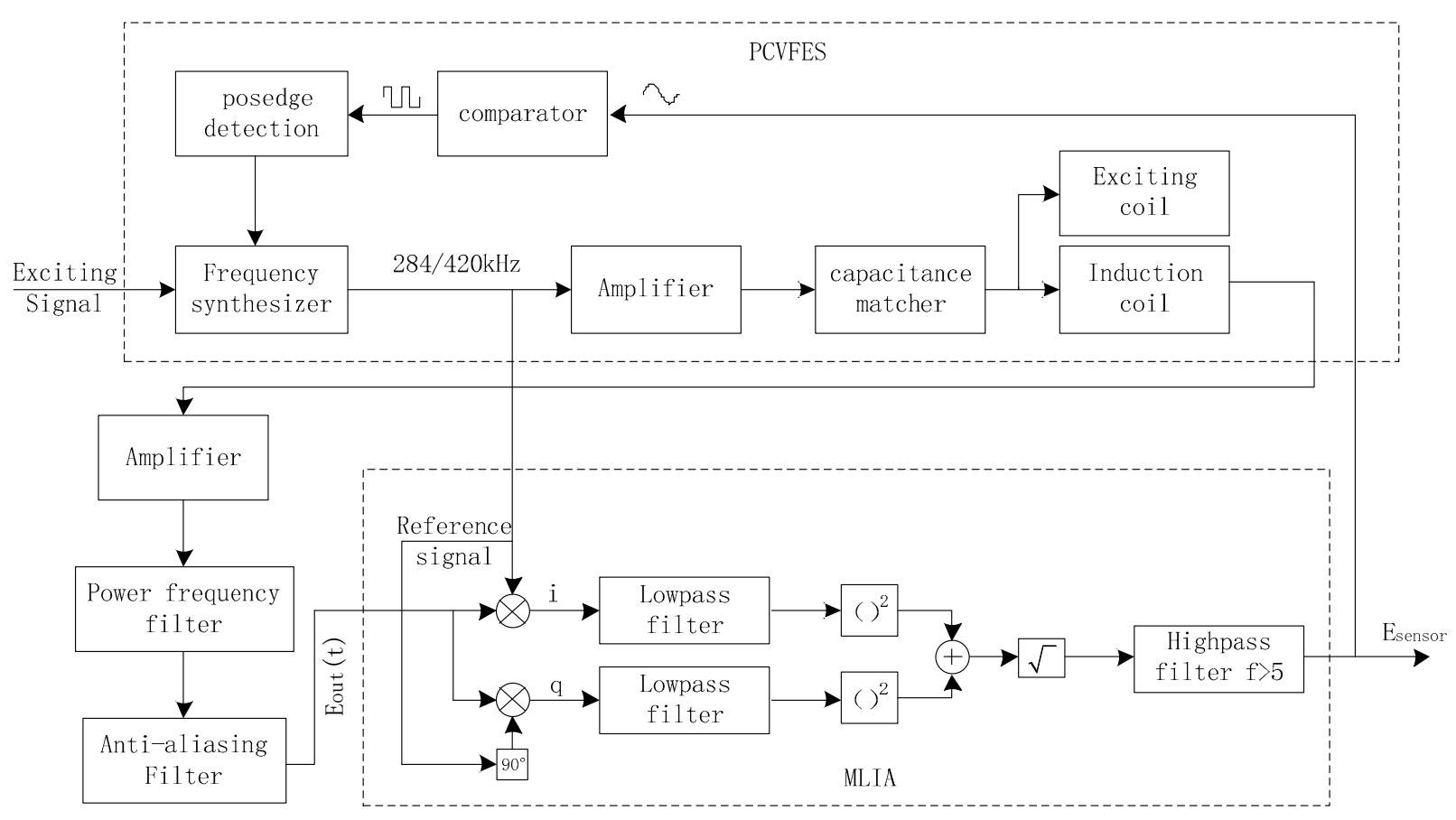

Fig.7 The block diagram of the signal exciting and detection system

\subsection{Application of the modified LIA on wear particles detection}

It is shown from theoretical analysis that the magnitude of induced electromotive force output by inductive coil is microvolt which has the same or smaller magnitude with the disturbance signal including interference signal caused by magnetic inhomogeneity and the background noises. Under an ideal condition, the inductive coil precisely lies in the middle of the structure, but because of some mismachining tolerance of coil substrate and the enwinding error of coils, the position of inductive coil must deviates the middle surface of the sensor. As a consequence, when no particles pass through sensor, the magnetic flow of the inductive coil is not equal to 0 , so the sinusoidal induced electromotive force generated at the initial stage which frequency is same with the exciting signal and the intensity closely related to the magnetic field inhomogeneity and offset of induction coil. Assuming the offset distance is $\Delta$, the initial induction electromotive force is $\mathrm{E}_{0}=\mathrm{E}(\Delta) \sin \left(\omega_{0} \mathrm{t}\right)$. In this circumstance, the initial induction electromotive force can be regarded as a carrier signal, and when a wear particle goes through the sensor, a new pulse signal will be generated because of the alteration of the magnetic flow inside the coils. The signal is $\mathrm{E}_{1}=\mathrm{E}\left(\mathrm{r}_{\mathrm{a}}, \mathrm{v}\right) * \sin \left(2 \pi \mathrm{f}_{1} \mathrm{t}\right)$ which amplitude is related to the radium of wear particles $r_{a}$ and the speed of passing the sensor $v, f_{l}$ is the frequency of particles passing through the sensor. In this sensor, the maximum quantity of flow was limited at $35 \mathrm{~L} / \mathrm{min}$, so the corresponding flow velocity is $v=15.16 \mathrm{~m} / \mathrm{s}$, and the frequency $\mathrm{f}_{1}=\mathrm{v} / \mathrm{l}_{1}=1.37 \mathrm{kHz}$. This signal will be amplitude modulated by the initial induction electromotive force $\mathrm{E}_{0}$, so the real signal output by the inductive coil is

$$
E_{\text {out }}=\left(E\left(r_{a}, v\right) * \sin \left(2 \pi f_{1} t+\varphi_{2}\right)+E(\Delta)\right) * \sin \left(\omega_{0} t+\varphi_{1}\right)+N(t)
$$

Where $\mathrm{N}(\mathrm{t})$ is Gaussian noise. We take a ferromagnetic particle as an example to analyze the detection principle of the valid signal using the MLIA, in which the real exciting frequency was set to $f_{0}$. So the reference signal is $\operatorname{Asin}\left(2 \pi f_{0} t+\varphi_{2}\right)$. The estimation procedure of the signal $E_{1}$ as follows:

At first, the real signal was multiplied by both the reference signal of Asin $\left(2 \pi f_{0} t+\varphi_{2}\right)$ and a signal in quadrature with respect to such a reference signal of $A \cos \left(2 \pi \mathrm{f}_{0} \mathrm{t}+\varphi_{2}\right)$. So the result after the MLIA's multipliers is: 


$$
\begin{aligned}
& i(t)=\left(\left(E\left(r_{\mathrm{a}}, v\right) * \sin \left(2 \pi f_{1}+\varphi_{2}\right)+E(\Delta)\right) * \sin \left(2 \pi f_{0} t+\varphi_{1}\right)+N(t)\right) A \sin \left(2 \pi f_{0} t+\varphi_{2}\right) \\
& =\frac{A}{2}\left(E\left(r_{\mathrm{a}}, v\right) * \sin \left(2 \pi f_{1}+\varphi_{2}\right)+E(\Delta)\right) * \cos \left(\varphi_{1}-\varphi_{2}\right)-\frac{A}{2}\left(E\left(r_{\mathrm{a}}, v\right) * \sin \left(2 \pi f_{1}+\varphi_{2}\right)+E(\Delta)\right) * \cos \left(2 * 2 \pi f_{0} t+\varphi_{1}+\varphi_{2}\right) \\
& +N(t) * A \sin \left(2 \pi f_{0} t+\varphi_{2}\right) \\
& q(t)=\left(\left(E\left(r_{\mathrm{a}}, v\right) * \sin \left(2 \pi f_{1}+\varphi_{2}\right)+E(\Delta)\right) * \sin \left(2 \pi f_{0} t+\varphi_{1}\right)+N(t)\right) * A \cos \left(2 \pi f_{0} t+\varphi_{2}\right) \\
& =\frac{A}{2}\left(E\left(r_{\mathrm{a}}, v\right) * \sin \left(2 \pi f_{1}+\varphi_{2}\right)+E(\Delta)\right) * \sin \left(\varphi_{1}-\varphi_{2}\right)+\frac{A}{2}\left(E\left(r_{\mathrm{a}}, v\right) * \sin \left(2 \pi f_{1}+\varphi_{2}\right)+E(\Delta)\right) * \sin \left(2 * 2 \pi f_{0} t+\varphi_{1}+\varphi_{2}\right) \\
& +N(t) * A \cos \left(2 \pi f_{0} t+\varphi_{2}\right)
\end{aligned}
$$

At this circumstance, the signal of the two channels $i(t)$ and $q(t)$ are made of three parts that are the amplitude component, high frequency part (frequency $=2 \mathrm{f}_{0}$ ) and the noise sector. After the LIA's low pass filters, the high frequency component and most of the noise signal can be removed through setting a suitable cut-of frequency for the low pass filters. Because the maximum value of $f_{1}$ is $1.37 \mathrm{kHz}$, so all the signals that frequency greater than $1.37 \mathrm{kHz}$ can be seen as invalid signal, so the cut-of frequency was set to $1.5 \mathrm{kHz}$, as a consequence, the following signals are acquired:

$$
\begin{aligned}
& i(t)=\frac{A}{2}\left(E\left(r_{\mathrm{a}}, v\right) * \sin \left(2 \pi f_{1}+\varphi_{2}\right)+E(\Delta)\right) * \cos \left(\varphi_{1}-\varphi_{2}\right) \\
& q(t)=\frac{A}{2}\left(E\left(r_{\mathrm{a}}, v\right) * \sin \left(2 \pi f_{1}+\varphi_{2}\right)+E(\Delta)\right) * \sin \left(\varphi_{1}-\varphi_{2}\right)
\end{aligned}
$$

In order to obtain the amplitude of the valid signal caused by wear particles, the two signals above mentioned $i(t)$ and $q(t)$ are calculated of their sum of squares and then extracted the square root. The estimation of the amplitude of particular frequency $\left(\mathrm{f}_{0}\right)$ component as follows:

$$
S C A=\sqrt{i(t)^{2}+q(t)^{2}}=\frac{A}{2}\left(E\left(r_{\mathrm{a}}, v\right) * \sin \left(2 \pi f_{1}+\varphi_{2}\right)+E(\Delta)\right)
$$

From the above equation, we can see that there are two components in the result that are sine wave element with a frequency of $f_{1}$ which represents the amplitude of the valid signal and the DC component which reflect the amplitude of the initial induction electromotive force. The $\frac{A}{2}$ indicates the magnification for the weak signal, in this system A equals to 5 that means the signal was amplified 2.5 times. At the same time, for the sake of the elimination of the DC component and availability of the valid weak signal, a high pass filter was arranged which cut-off frequency was set to $5 \mathrm{~Hz}$ to guarantee the availability of the sinusoidal frequency component in the signal. So the weak induction electromotive force can be obtained that:

$$
E_{\text {out }}=\frac{A}{2}\left(E\left(r_{\mathrm{a}}, v\right) * \sin \left(2 \pi f_{1}+\varphi_{2}\right)\right.
$$

It is noteworthy that based on the characteristic of this system the $\mathrm{f}_{1}$ should be greater than $5 \mathrm{~Hz}$ otherwise it will also be eliminated, which means the minimum velocity of the wear particles through the sensor must larger than $\mathrm{v}=\mathrm{f}_{1} * 1_{1}=5 * 11 \mathrm{e}-3=5.5 \mathrm{e}-2 \mathrm{~m} / \mathrm{s}$, and the corresponding quantity of flow is $v \frac{\pi}{4} d^{2}=0.127 L /$ min . Assuming the speed of the particle movement is equal to the fluid velocity, the acceptable flow of the sensor ranges from $0.127 \mathrm{~L} / \mathrm{min}$ to $35 \mathrm{~L} / \mathrm{min}$.

In order to validate the detectability of the weak induced electromotive force using the proposed method, signals simulated in MATLAB, the initial induction electromotive force was set to $\mathrm{E}_{0}=5 \mathrm{e}-4 \sin \left(\omega_{0} \mathrm{t}\right)$, and we take a 
more faint signal $E_{1}=5 e-5 * \sin \left(2 \pi f_{1}+\varphi_{2}\right)$ to represent the valid induced electromotive force induced by wear particles. At the same time a Gaussian noise was also added to the signal which variance is $1 \mathrm{e}-8$. So the simulation signal is based on the following equation:

$$
E_{\text {out }}=\left(5 e-5 * \sin \left(2 \pi f_{1}+\varphi_{2}\right)+5 e-4\right) \sin \left(2 \pi f_{0}+\varphi_{1}\right)+N(t)
$$

Where $\mathrm{f}_{0}$ and $\mathrm{f}_{1}$ were set to $284 \mathrm{kHz}$ and $1.37 \mathrm{kHz}$ respectively. From the above equation we can obtain that the amplitude of the valid induced electromotive force would be at least an order of magnitude lower than that of initial induced electromotive force caused by coil offset and the Gaussian noise. So it is easily disturbed by these invalid signals.

According to the above simulation parameters we can obtain that the main components in the real signal are the Gaussian noises and initial induced electromotive force, while the amplitude of the induced electromotive force caused by wear particles is really smaller than others. Fig.8a shows the waveform of the original signal, so it is very hard to directly recognize the valid signal. While after the MLIA system the valid signal hidden in the interference signals was successfully extracted as Fig. 8 b.

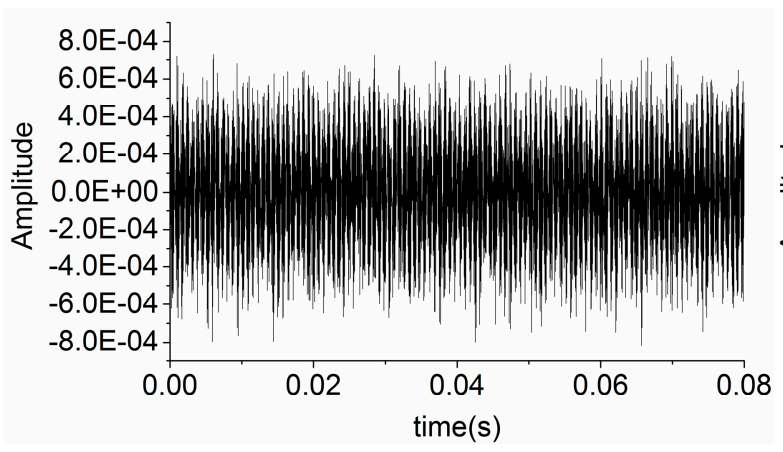

(a)

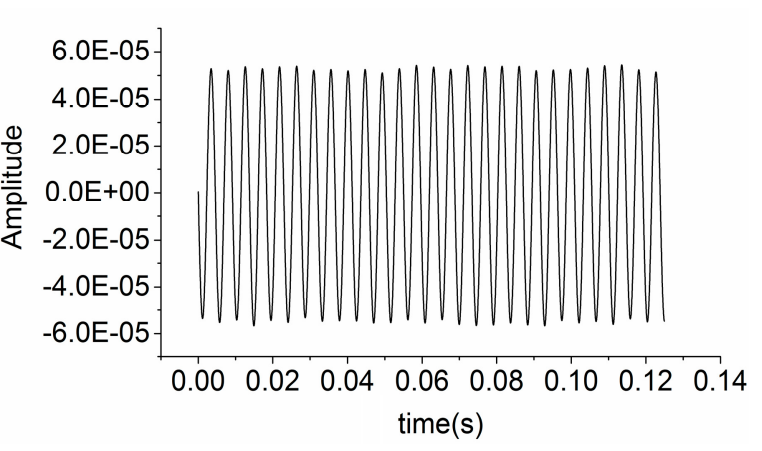

(b)

Fig.8 Simulation signal (a) the real signal and (b) the result of the detection

\section{Experiment results and discussions}

\subsection{Experiment system}

The complete experimental system is shown in the Fig.9a which consist the sensor shown in Fig.9b, the Excitation and detection unit, the Data collecting and processing software written in $\mathrm{C}++$ language. The specially sized abrasive particles are fed into the sensor in batches to certify the sensitivity and the detection ability of micro wear particles. In the experiment, iron particles and copper particles of different particle sizes are used. Since the effect of the same volume of iron particles on the magnetic field is much greater than that of the copper particles on the magnetic field, so the diameters of the selected iron particles are $75 \mu \mathrm{m}, 100 \mu \mathrm{m}, 120 \mu \mathrm{m}, 150 \mu \mathrm{m}$, and for copper particles are $220 \mu \mathrm{m}, 280 \mu \mathrm{m}$ and $380 \mu \mathrm{m}$. Some sphere-like wear particles that meet the above diameter requirements were selected by the scanning electron microscope. The selected abrasive particles are shown in the Fig.10.Before the experiment, the responses of the sensor were compared between situations of wear particles in the oil and oil-free environment, and the results difference in both cases is tiny, so all the test were conducted in the absence of fluid to measure the output response of the sensor. 


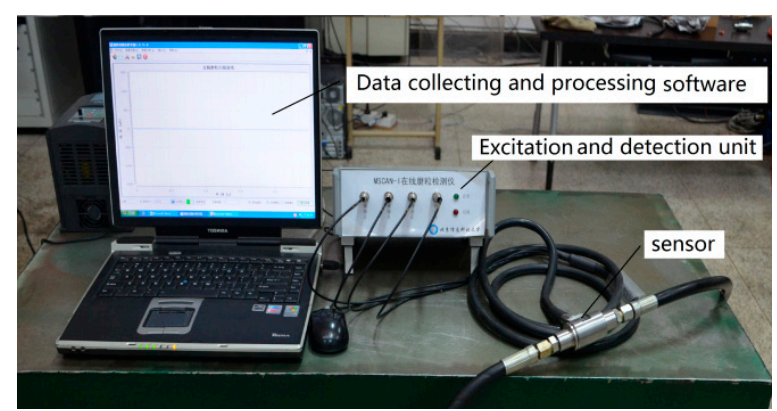

(a)

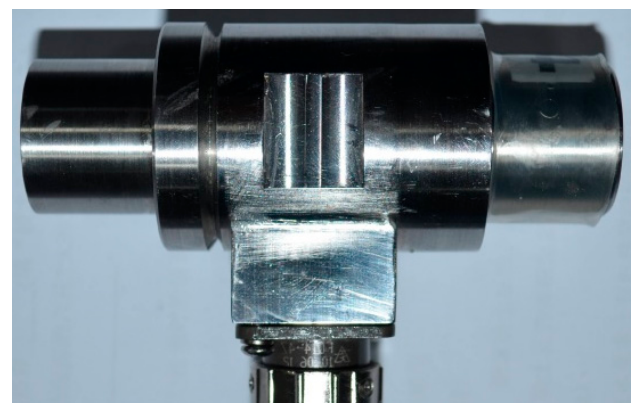

(b)

Fig.9 The experiment system
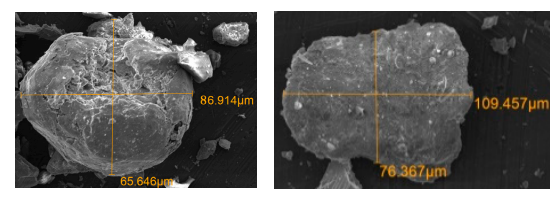
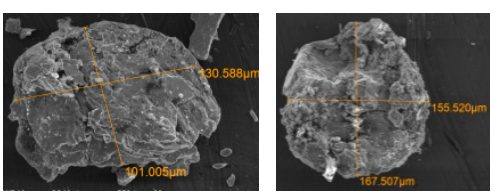

(a)
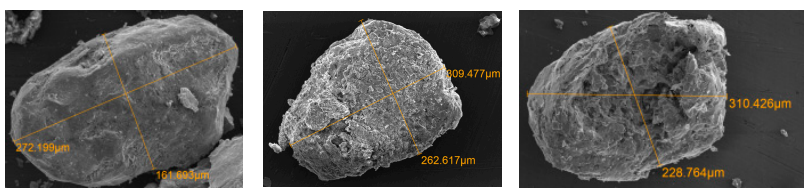

(b)

Fig. 10 Wear particles (a) Iron particles and (b) Copper particles

\subsection{Effectiveness of the MLIA system}

The effectiveness of modified LIA system was also verified by real output signal of the sensor. Experiment was carried out using a $100 \mu \mathrm{m}$ iron particle as the detection object and compared the original signal of the induction coil and the detection result after the MLIA system. By measuring the initial induced electromotive force output due to the magnetic field imbalance of the two exciting coils is $E(\Delta)=3.2 \mathrm{e}-3 \sin \left(2 \pi \mathrm{f}_{0} \mathrm{t}\right)$, when the iron particle access to the sensor, the real signal comes from induction coil is shown at Fig.11a.It is obvious that the majority of the signal is the noises and initial inductive electromotive force, it is So difficult to directly observe the valid signal caused by the iron particle. After the MLIA system, the disturbance signals are all filtered and valid signal is successfully extracted as Fig.11b.

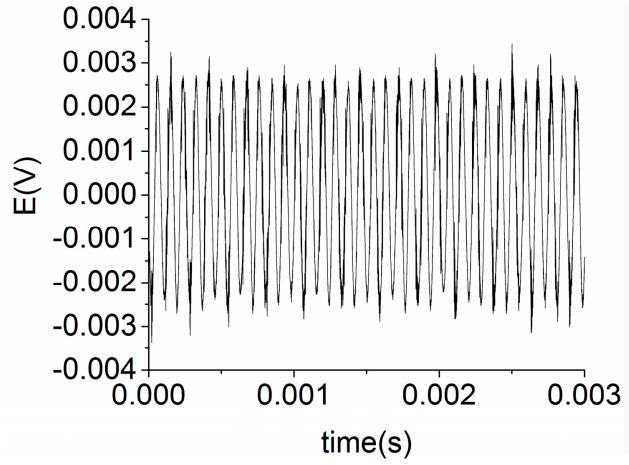

(a)

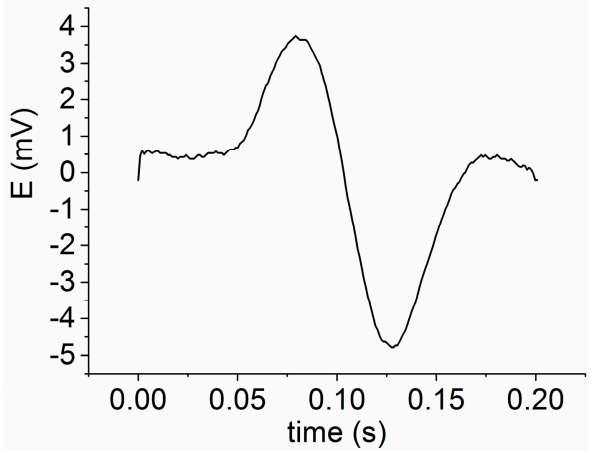

(b)

Fig. 11 Effect of the MLIA system

\subsection{Sensitivity comparison for ferromagnetic particles detection}

From the Figs. 4,5 the decrease of resonance capacitance does enhance the impedance change of exciting circuit caused by micro particles and further increase the current difference between two exciting coils and 
eventually improve the sensitivity of the sensor. In the experiment, a series of 2-10nF capacitors were used to verify the sensitivity changes of the sensor as Fig.12. We can easily obtain that as the excitation capacitance decreases, the sensor sensitivity increases gradually. In the circumstance of resonance capacitance equals to $2 \mathrm{nF}$, the corresponding resonance frequency is $284 \mathrm{kHz}$; the resonance capacitance for inductance coil is $5.45 \mathrm{nF}$. In this case the signal amplitude caused by an iron particle of diameter $75 \mu \mathrm{m}$ is $7.9 \mathrm{mV}$ which is much greater than the voltage value $(3.2 \mathrm{mV})$ of the circumstance $\mathrm{C} 1=\mathrm{C} 2=10 \mathrm{nF}$, and the increasing trend tends to be more evident for larger particles. But simply reducing the resonance capacitance will lead to the decrease of sensitivity for ferromagnetic debris due to the increase of eddy current effect in the particle, so the final resonant capacitor of exciting coil was selected as $2 \mathrm{nF}$ for ferromagnetic particles detection.

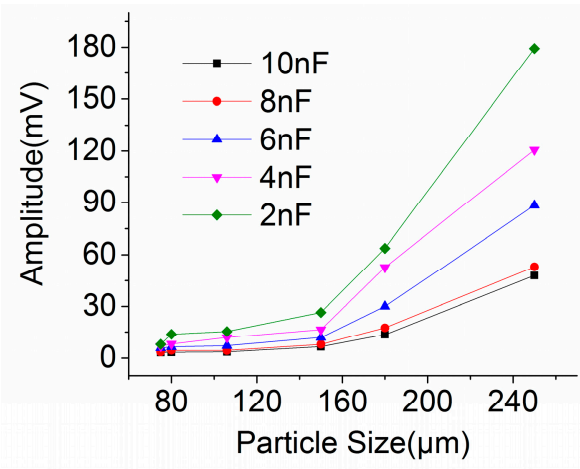

Fig.12 sensitivity comparison

In order to further describe the role of resonance principle on sensitivity improvement, the sensors with LC resonance method (see figure 2(b)) and non-resonance method (see figure 2(a)) were tested. Figure 13 shows the output signal of the sensors when the four different sizes of iron particles pass through it. In the figure, the red curve illustrates the voltage change output by sensor (a) with non-resonance method and the black curve represents the output of the sensor (b) with resonance principle. It can be seen that the difference of output signal between the two sensors is very significant. For the conventional sensor, it hard to successfully detect iron particles with diameter less than $100 \mu \mathrm{m}$, meanwhile, the induced electromotive force caused by an iron particle $100 \mu \mathrm{m}$ in diameter is just $2.25 \mathrm{mV}$. However for the sensor with resonance principle, it can easily detect the iron particles with a diameter of $75 \mu \mathrm{m}$ and the amplitude of signal can reach to $7.9 \mathrm{mV}$. The comparison about the sensitivity of the two sensors is shown in Fig.15. The results clearly show that the sensitivity of the sensor with LC resonance method is more than three times than the sensor with non-resonance method. Meanwhile the detection capability for micro particles of the sensor has been improved due to the increase of the sensitivity.

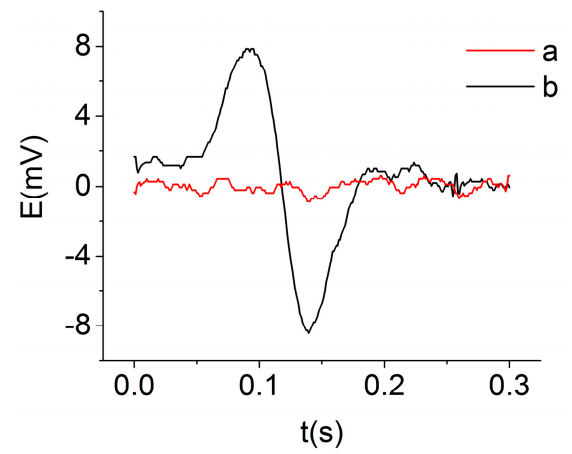

(a) $75 \mu \mathrm{m}$

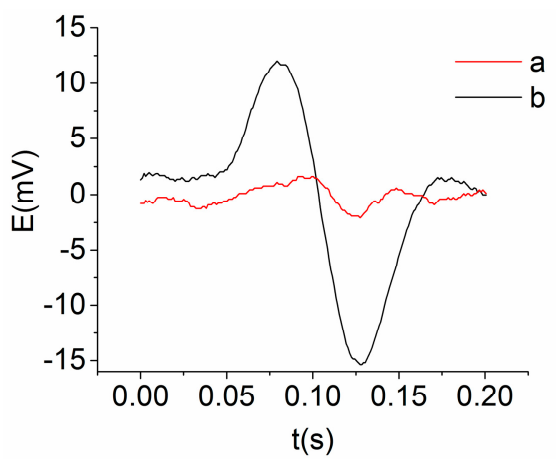

(b) $100 \mu \mathrm{m}$ 


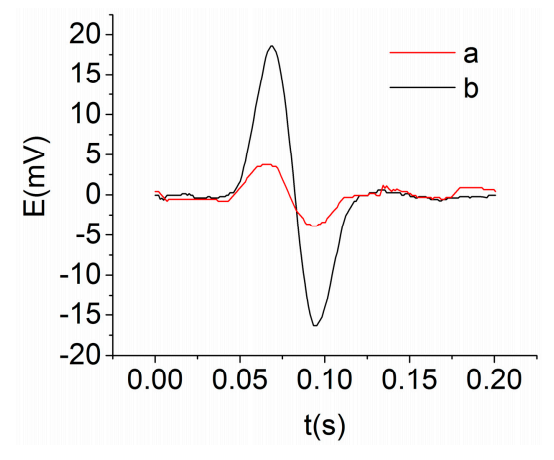

(c) $120 \mu \mathrm{m}$

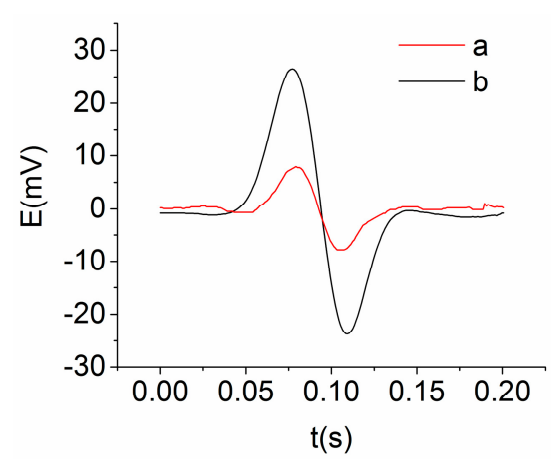

(d) $150 \mu \mathrm{m}$

Fig.13 The induced electromotive force caused by iron particles

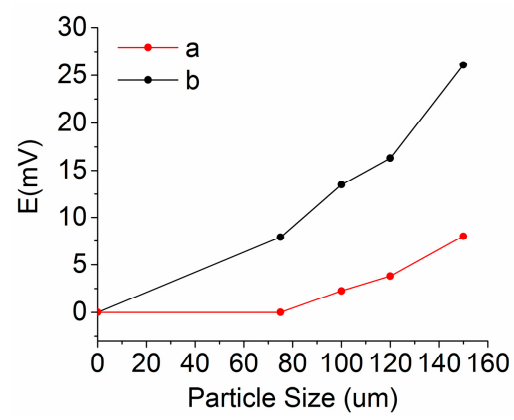

Fig.14 The comparison of sensitivity for iron particle detection

\subsection{Sensitivity comparison for non-ferromagnetic particles detection}

For the detection of the non-ferromagnetic particles, the principle is mainly based on eddy current effect which is closely related to the exciting frequency. Therefore in the detection process of non-ferromagnetic debris, increasing the excitation frequency will both enhance the eddy current effect in the particle and improve the sensitivity of the sensor. But nevertheless the influence of the same volume of non-ferromagnetic particles on the magnetic field is still much smaller than that of ferromagnetic particles. So in the experiment, the copper particles with diameter of $220 \mu \mathrm{m}, 280 \mu \mathrm{m}$ and $380 \mu \mathrm{m}$ were adopted. In order to improve the detection ability for non-ferromagnetic particles, the exciting frequency was further increased to $420 \mathrm{kHz}$. It is worth stressing that the exciting frequency is automatic switched between $284 \mathrm{kHz}$ and $420 \mathrm{kHz}$ based on the phase of induced electromotive force by the PCVFES and the resonant capacitor is also automatically adjusted by the capacitance matcher. Through the experiment we found that it is difficult to effectively detect the above copper debris in the circumstance of exciting frequency smaller than $300 \mathrm{kHz}$. Fig. 15 illustrates the output signal of the sensor with LC resonance method for copper particles detection at the situation of exciting frequency was set to $315 \mathrm{kHz}(1.6 \mathrm{nF})$ and $420 \mathrm{kHz}(0.9 \mathrm{nF})$. It shows that the sensitivity of the sensor has been improved with the increase of exciting frequency.

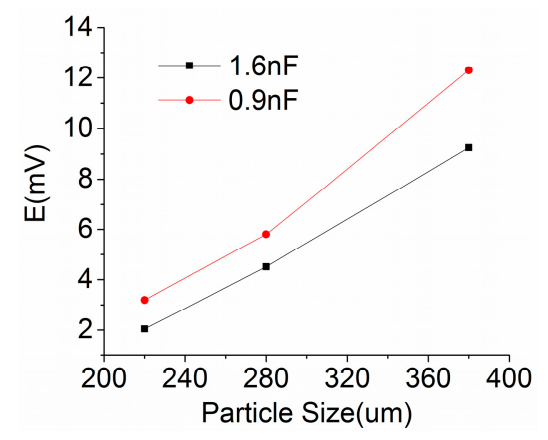

Fig.15 Parameters of the sensor 
The experiment also verified the sensitivity difference of the two types of sensors for non-ferromagnetic particles detection at the circumstance of exciting frequency equals to $420 \mathrm{kHz}$. Figure 16 shows the output signal of the sensors when the three different sizes of copper particles enter into it. It can be seen that the conventional sensor completely failed in the detection process of $220 \mu \mathrm{m}$ copper particles. And for the larger particles, the induced electromotive force is also very weak; the amplitude of signal caused by the copper particle $380 \mu \mathrm{m}$ in diameter is just $2.1 \mathrm{mV}$. In comparison, the detection ability of the sensor with resonance method is greatly improved; the peak of the signal caused by the three copper particles is about $3.2 \mathrm{mV}, 5.8 \mathrm{mV}$ and $12.3 \mathrm{mV}$. The comparison about the sensitivity of the two sensors for copper particle detection is shown in Fig.17. The result shows that introducing the resonance method into the sensor and further increasing the exciting frequency to 420 $\mathrm{kHz}$ will enhance the sensitivity of the sensor more than 4times as original for the non-ferromagnetic particles detection.

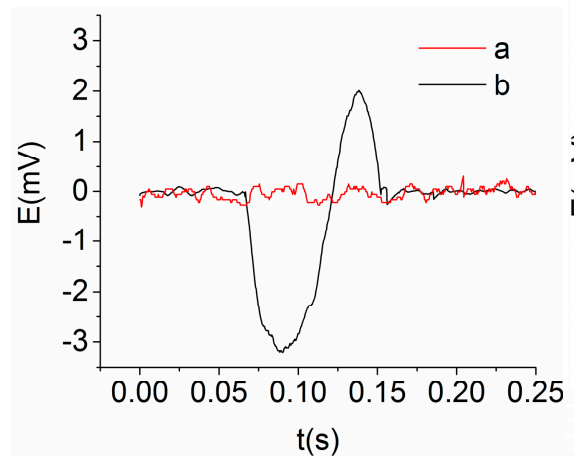

(a) $220 \mu \mathrm{m}$ (Copper)

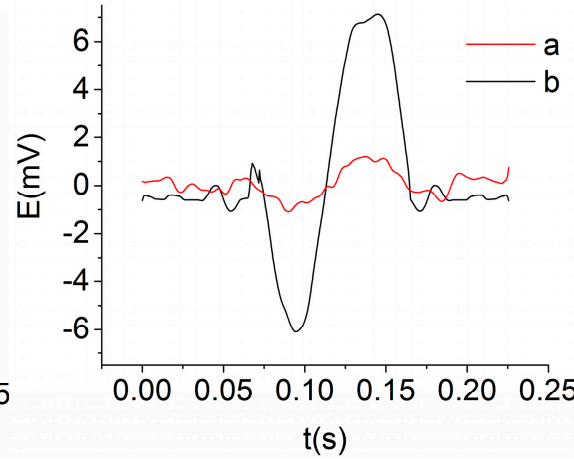

(b) $280 \mu \mathrm{m}$ (Copper)

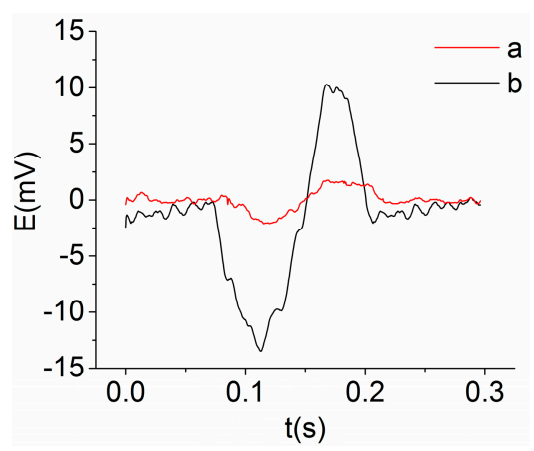

(c) $380 \mu \mathrm{m}$ (Copper)

Fig.16 Parameters of the sensor

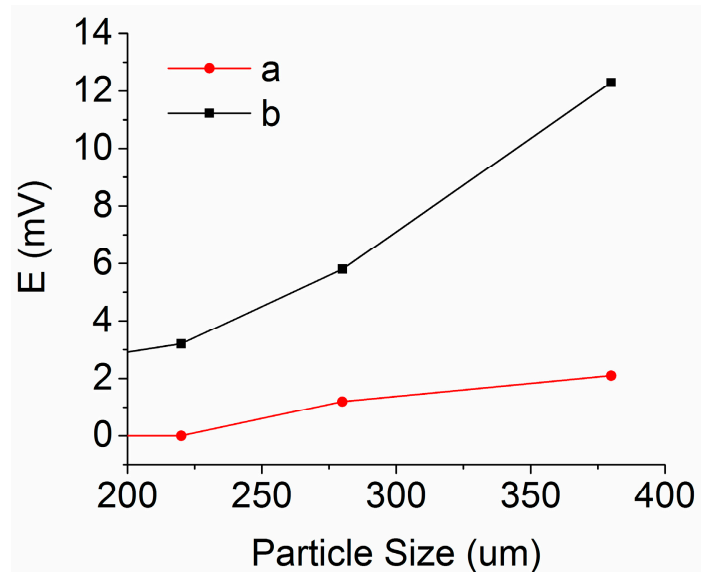

Fig.17 The comparison of sensitivity for copper particle detection

\section{Conclusion}

In order to meet the requirements of on-line wear status monitoring of heavy vehicles we studied the sensitivity enhancement method and the extraction method for weak induced electromotive force of the inductive wear monitoring sensor with large aperture $(7 \mathrm{~mm})$. We introduced the LC resonance principle into the three-coil inductive wear monitoring sensor and make all the coils working in the resonance status simultaneous. At this circumstance, the impedance change of exciting circuit is much larger than the exciting coil impedance change when wear particles pass through the sensor which will magnify the current difference between the both sides of exciting coils and cause a greater magnetic flux change in the induction coil and finally lead to the sensitivity improvement of sensor. At the same time, by reducing the resonance capacitance and increasing the exciting frequency to further improve the sensitivity and the detection ability for Micro particles, but the excessive exciting 
frequency will weaken the detection performance for ferromagnetic particles while boost the detection performance for non-ferromagnetic particles, So the PCVFES was designed to automatically switch the exciting frequency between $284 \mathrm{KHz}$ and $420 \mathrm{KHz}$ for ferromagnetic and non-ferromagnetic particles respectively. The resonant induction coil has a higher capability of noise reduction and signal magnification which will further improve the sensitivity of the sensor. About the signal detection method, unlike other researcher using mathematical algorithm for example EMD and Wavelet Transform to extract the signal characteristics, however, a simpler and more effective method that the modified lock-in amplifier was adopted based on the essential nature of the signal that composed of Gaussian noise, initial induced electromotive force caused by magnetic inhomogeneity and the weak signal result from wear particles. The MLIA system can be implemented entirely through hardware which greatly reduce the amount of calculation of the system and improve the real-time performance of the sensor. Through the LC resonance method, variable excitation frequency system and weak signal detection system, the sensor can eventually achieve the effective detection for the $75 \mu \mathrm{m}$ ferromagnetic particles and $220 \mu \mathrm{m}$ non-ferromagnetic particles which meets the needs of on-line wear status monitoring of vehicles and other engineers with large flow, and can effectively monitor the abnormal wear of the mechanical system at the initial stage.

\section{Acknowledgments}

This work was supported by National Natural Science Foundation of China (NSFC) (grant number:51475044) and Beijing finance found of science and technology planning project (grant number: KZ201611232032).

\section{Author Contributions}

Authors Jia Ran, Ma Biao and Zheng Changsong proposed and developed the system construction method, theoretical analysis and simulation, Jia Ran, Wang Liyong and Du Qiu carried out all the experiment of the research. JiaRan wrote this paper under supervision of professor MaBiao and Zheng Changsong.

\section{Conflicts of Interest}

The authors declare no conflict of interest.

\section{Reference}

[1] Loutas, T.H.; Roulias, D.; Pauly, E.; Kostopoulos, V. The combined use of vibration, acoustic emission and oil debris on-line monitoring towards a more effective condition monitoring of rotating machinery. Mech. Syst. Signal Process. 2011, $25,1339-1352$.

[2] L. Du, J. Zhe, Parallel sensing of metallic wear debrisin lubricants using under sampling data processing,Tribology International, Vol. 53, 2012, pp. 28-34.

[3] H. B. Fan, Y. T. Zhang, G. Q. Ren, Z. N. Li,Experimental Study of an On-line Monitoring Sensorfor Wear Particles in Oil, Tribology, Vol. 30, No. 4, [1] 2010, pp. 338-343.

[4] YH Yin; XP Yan; HL Xiao; CT Wang. Design of the inductive transducer of wear particle monitoring [J]. Journal of Transducer Technology, 2003, 07:

[5] Du L, Zhe J, Carletta J E, et al. TECHNICAL NOTE:, Inductive Coulter counting: detection and differentiation of metal wear particles in lubricant[J]. Smart Materials \& Structures, 2010, 19(5):057001.

[6] Zhan H, Song Y, Zhao H, et al. Study of the Sensor for On-line Lubricating Oil Debris Monitoring[J]. Sensors \& Transducers, 2014, 175(7): 214.

[7] EC Liu, HP Zhang, XR Zhang, et al. Dual-coil solenoid sensor design and its experimental study for wear particles detection [J]. Journal of Dalian Maritime university ,2016,(02):102-106+116.

[8] Han L, Hong W, Wang S. The Key Points of Inductive Wear Debris Sensor[C]// International Conference on Fluid Power and Mechatronics. 2011:809-815. 
[9] Yin, Y.; Wang, W.; Yan, X.; Xiao, H.; Wang, C. An integrated on-line oil analysis method for condition monitoring. Meas. Sci. Technol. 2003, 14, 1973-1977.

[10] Kim B, Han S H, Kim K. Planar Spiral Coil Design for a Pulsed Induction Metal Detector to Improve the Sensitivities[J]. IEEE Antennas \& Wireless Propagation Letters, 2014, 13:1501-1504.

[11] Davis J P, Carletta J E, Veillette R J, et al. Instrumentation circuitry for an inductive wear debris sensor[C], New Circuits and Systems Conference (NEWCAS), 2012 IEEE 10th International. IEEE, 2012: 501-504.

[12] Xing-ming Z, Hong-peng Z, Zhao B O, et al. Study On Magnetization and Detection the Metal Particle in Harmonic Magnetic Field[J]. Key Engineering Materials, 2015.

[13] Vutha A K, Davaji B, Lee C H, et al. A microfluidic device for thermal particle detection[J]. Microfluidics \& Nanofluidics, 2014, 17(5):871-878.

[14] Du L, Zhu X, Han Y, et al. Improving sensitivity of an inductive pulse sensor for detection of metallic wear debris in lubricants using parallel LC resonance method[J]. Measurement Science \& Technology, 2013, 24(7):660-664.

[15] Li, C.; Liang, M. Extraction of oil debris signature using integral enhanced empirical mode decomposition and correlated reconstruction. Meas. Sci. Technol. 2011, 22, 085701.

[16] Li C, Peng J, Liang M. Enhancement of the wear particle monitoring capability of oil debris sensors using a maximal overlap discrete wavelet transform with optimal decomposition depth[J]. Sensors, 2014, 14(4):6207.

[17] Li, J.S.; Parchatka, U.; Fischer, H. Applications of wavelet transform to quantum cascade laser spectrometer for atmospheric trace gas measurements. Appl. Phys. B 2012, 108, 951-963.

[18] Yi, T.H.; Li, H.N.; Zhao, X.Y. Noise smoothing for structural vibration test signals using an improved wavelet thresholding technique. Sensors 2012, 12, 11205-11220.

[19] Bonnet, V.; Ramdani, S.; Azevedo-Coste, C.; Fraisse, F.; Mazzà, C.; Cappozzo, A. Integration of human walking gyroscopic data using empirical mode decomposition. Sensors 2014, 14, 370-381.

[20] Braun, S.; Feldman, M. Decomposition of non-stationary signals into varying time scales: Some aspects of the EMD and HVD methods. Mech. Syst. Signal Process. 2011, 25, 2608-2630.

[21] Z Gao, CS Zheng, R Jia, XP Zhang, ZQ Liu, JH Fan. Study on-line monitoring wear debris sensor of power shift-steering transmission [J]. Journal of Guangxi University (Nat Sci Ed),2017,(02):409-418 